\title{
SELF-DUAL AXIOMS FOR MANY-DIMENSIONAL PROJECTIVE GEOMETRY
}

BY

\section{MARTINUS ESSER}

\begin{abstract}
Proposed and compared are four equivalent sets $R, S, T, D$ of self-dual axioms for projective geometries, using points, hyperplanes and incidence as primitive elements and relation. The set $R$ is inductive on the number of dimensions. The sets $S, T, D$ all include the axiom "on every $n$ points there is a plane", the dual of this axiom, one axiom on the existence of a certain configuration, and one or several axioms on the impossibility of certain configurations. The se configurations consist of $(n+1)$ points and $(n+1)$ planes for sets $S, T$, but of $(n+2)$ points and $(n+2)$ planes for set $D$. Partial results are obtained by a preliminary study of self-dual axioms for simplicial spaces (spaces which may have fewer than 3 points per line).
\end{abstract}

1. Definitions and results. Projective spaces, with finite dimension $n$, are defined classically by nondual axioms, using undefined points and certain sets called lines. We propose several equivalent sets of self-dual axioms, using undefined points, planes (= hyperplanes) and incidence. With further details and definitions to be given later, these axioms for Projective spaces $P$ [or in brackets for simplicial spaces $N]$ are

The classic $[1$, p. 24] Set $L P$ [or $L N]$.

Axiom I. Any two distinct points are in exactly one line.

Axiom IIP [or IIN]. There exist at least three [or two] points in each line.

Axiom III. If five distinct points $P, Q, R, S, T$ satisfy the collineations $\overline{P Q R}$, $\overline{P S T}$, then there exists a point $U$ satisfying $\overline{Q S U}, \bar{R} \overline{T U}$.

Axiom IV (and V). There exists at least (and at most) $n+1$ independent points.

A Set $R P$ [or $R N]$ inductive on dimensions.

Axiom a. For $n=1$ the relation "on" is one-to-one.

Axiom bP[or bN]. For $n=1$ there exist at least three [or two] points.

Axiom c. For $n \geq 2$ there exist at least one point and one plane not on each other.

Axiom d. For $n \geq 2$ any reduction $[R, r]$ is an $(n-1)$-dimensional space.

Presented to the Society, November 3,1969; received by the editors February 5, 1971 and, in revised form, February 3, 1972.

AMS (MOS) subject classifications (1970). Primary 50A10; Secondary 05B25, 50D35.

Key words and phrases. Projective, geometry, axioms, designs (or blocks), foundations. 
A Set TP [or $T N]$ of axioms by configurations.

Axiom 1 (and 2). On every $n$ points (planes) there exists at least one plane (point).

Axiom 3TP[or 3TN]. There exists a semidoublex [or a semis implex].

Axiom 4 (and 5). Distinct planes (points) are distinguishable.

Axiom 6T. If $T$ is a point on the $n$ planes, and if $t$ is a plane on the $n$ points, of a semisimplex, then $T$ is not on $t$.

If we replace Axiom $3 T P$ [or $3 T N$ ] by the stronger

Axiom 3D[or 3SN]. There exists a doublex [or a simplex], then we shall replace Axioms 4, 5, 6T by weaker axioms given later.

We consider also Weak spaces $W$ defined equivalently by later configuration axioms or by the following inductive Set $R W$.

Axiom aW. For $n=0$ all incidences are "not on".

Axioms bW and $\mathrm{cW}$. For $n=0$ and for $n \geq 1$ there exist a point and a plane not on eacb other.

Axiom dW. For $n \geq 1$ any reduction $[R, r]$ is an $(n-1)$-dimensional space.

We repeat that we start with undefined objects called points, undefined objects called planes, and an undefined relation on between single points and single planes. The symbols $\circ$ and $\sim$ mean "on" and "not on". Thus $A \circ a, A \sim b$ means that the point $A$ is on the plane $a$ but not on the plane $b$. Independent is defined in $\S 5$. By a reduction $[R, r]$, where always we assume $R \sim r$, we mean the set of all points on the plane $r$ and of all planes on the point $R$. Two planes (or points) are said to be distinguishable if there exists a point (or plane) on exactly one of the two planes (or points). Duality means to interchange points and planes.

Axioms and configurations are identified by letters: $P=$ projective, $N=$ simplicial, $W=$ weak, $U=$ undistinguished, $L=$ lines, $R=$ reduction, $S=$ square, $T=$ triangle, $D=$ doublex. For any positive integer $\alpha$, a set of $\alpha$ points $A i$ and of $\alpha$ planes $a i$, with $i=1,2, \cdots, \alpha$, is called an $\alpha$-square if the corresponding (square) incidence table is completely specified, and is called an $\alpha$-triangle if the incidences $A i$ ? $a j$ are specified for at least all $i \geq j$. We define various configurations (giving notation $=$ name $=$ properties)

$T N \alpha=$ singlenot $\alpha$-triangle $=\alpha$-triangle with $A i \circ$ aj for $i>j ; A i \sim a i$.

$S N \alpha=$ singlenot $\alpha$-square $=\alpha$-square with $A i \circ a j$ for $i \neq j ; A i \sim a i$.

$T P \alpha=$ doublenot $\alpha$-triangle $=\alpha$-triangle with $A i \circ a j$ for $i>j ; A i \sim a i, A i \sim$ $a(i+1)$.

$S P \alpha=$ doublenot $(x-s q u a r e=\alpha$-square with $A i \sim a i, A i \sim a(i+1) ; A i \circ a j$ otherwise.

$D(n+2)=$ doublex $=(n+2)$-square with $A i \sim a i, A i \sim a(i+1), A(n+2) \sim$ $a 1 ; A i \circ a j$ otherwise. 
In particular

Semisimplex $=T N n$, Simplex $=S N(n+1)$, Semidoublex $=T P(n+1)$.

It is allowed to take $\alpha=1$ in a doublenot triangle or square, the hypothes is $A i \sim a(i+1)$ being then vacuously true. Indeed for $\alpha=1$ we have $T N 1=S N 1=$ $T P 1=S P 1$. We use capitals for points, lower cases for planes, script capitals for squares. For instance the $\alpha$-square $\mathscr{B}$ has automatically the points $B i$ and the planes $b i$ with $1 \leq i \leq a$.

The axioms by configurations, including axioms given earlier, and to be considered in various later sets, are

Axioms 3TN, 3SN, 3TP, 3SP or 3D. There exists a configuration $T N n$, $S N(n+1), T P(n+1), S P(n+1)$ or $D(n+2)$ respectively.

Axiom 4N. There is only one plane on the first $n$ points of any simplex.

Axioms 6T, 6W or 6P. If a point $T$ is on the $n$ planes, and if a plane $t$ is on the $n$ points, of a configuration $T N n, S N n$ or $S P n$ respectively, then $T \sim t$.

Axiom 6D. Doublenot $(n+2)$-squares are impossible.

Axioms 7N, 7P or 7D. No plane is on the $(n+1)$ points of a configuration $S N(n+1), S P(n+1)$ or on the $(n+2)$ points of a doublex $D(n+2)$.

Axioms 5N, 8N, 8P or 8D. Duals of Axioms $4 N, 7 N, 7 P, 7 D$.

We consider the following sets of axioms:

$L N, L P, R W, R N, R P$ defined earlier,

$T N=\{1,2,3 T N, 4,5,6 T\}$,

$T U=\{1,2,3 T P, 6 T\}, T P=\{T U, 4,5\}$,

$S W=\{1,2,3 S N, 6 W\}$,

$S N=\{1,2,3 S N, 4 N, 5 N\}$,

$S U=\{1,2,3 S P, 7 P, 8 P\}, S P=\{S U, 4,5\}$,

$D U=\{1,2,3 D, 6 D, 7 D, 8 D\}, D P=\{D U, 4,5\}$.

Instead of Set $X$ of axioms, often we write Axioms $\{X\}$. The Axioms $6 P, 7 N$, $8 \mathrm{~N}$ do not appear in any previous set, but will be used in proofs.

We shall prove

Theorem Al. Sets RW and SW are equivalent (and define weak spaces). Proof in $\$ 3$.

Theorem Bl. Sets $R N, S N, T N, L N$ are equivalent (and define simplicial spaces). Proof in $\$ \$ 3,4,5$.

Theorem Cl. Sets TU, SU, DU are equivalent. Proof in $\$ \$ 6,8$.

Theorem Dl. Sets $L P, R P, T P, S P, D P$ are equivalent (and define projective spaces). Proof in $\$ \$ 5,6,8$.

In the last section we study some examples, and discuss independencies within sets of axioms. 
Theorem $\mathrm{D}$ is our main achievement. Its proof requires all results in this article, including the the ory of $W$ and $N$ spaces. The longest proofs are Set $T P \Rightarrow$ Set $R P$ and Set $D U \Longrightarrow$ Axiom $6 T$. The se two proofs have a large common part, in particular $\$ \$ 2$ and 7 . The Axioms 4,5 have no interest in discussions involving only configuration axioms (because an identification of undistinguishable elements is trivial), but they are fundamental when studying reduction axioms (because distinguishability in the whole space does not imply distinguishability in the reductions).

We have also

Theorem E1. Any $(n+2)$ points are the points of a doublex if and only if no $(n+1)$ of them are coplanar.

Proof. The proof is easy. Axiom 1 implies the "if" and Lemma B7 gives the "only if".

Theorem F1. For two-dimensional spaces Axioms 1, 2, 3TP, 6P imply Axiom $6 T$.

Proof. The proof of this innocuous theorem is difficult and involves most results developed in this article. See Theorem A9. The proof given there shows that Axioms $(1,2,3 T P, 6 P) \Rightarrow$ Axiom $3 S P$ for $n=2$ only, and that Axioms $(1,2,3 S P, 6 P) \Rightarrow$ Axiom $6 T$ for all $n$.

The Set $S N$ appeared already in [2] and the set $(1,2,3 D, 6 T)$, for $n=3$ only, appeared in [5].

2. Moving a simplex. We assume Axioms $\{S W\}$ or $\{S N\}$ and prove

Theorem A2. For every point $R$ and plane $r$ with $R \sim r$ there exists a simplex B such that $B(n+1)=R, b(n+1)=r$.

This theorem states the existence of a simplex $\mathcal{B}$ containing a given set $\mathcal{S}$ of points and planes. We find $B$ by "moving" a simplex from the starting position $\mathfrak{A}$, which is the simplex of Axiom $3 S N$, into the desired terminal position $B$, by successive replacements of one point or one plane of $\mathfrak{A}$ by one point or one plane of $\mathfrak{S}$.

The notation $\{\ldots \uparrow \ldots\}$ means the finite sequence starting with the term on the left and ending with the term on the right. Thus $\{B 6 \uparrow B 8\}=\{A 2\lceil A 4\}$ means $B 6=A 2, B 7=A 3, B 8=A 4$.

Lemma B2. A permutation of the points of a simplex and the same permutation of the planes yields a new simplex.

The proof is obvious.

Lemma C2. No plane is on all points of a simplex (reworded: Axiom $6 W$ or $4 N \Rightarrow$ Axiom $7 N$ ). 
Proof. The existence of a plane $t$ on all points of a simplex $\mathbb{Q}$ contradicts Axiom $6 W$ with $T=A(n+1), t=t, S N n=\left\{A 1 \uparrow A n ; a 1 \uparrow a_{n}\right\}$, and contradicts Axioms $4 N$ because $t \neq a(n+1)$.

Given a simplex $\mathbb{Q}$, a point $P$, and two integers $\alpha, \beta$ with $1 \leq \alpha<\beta \leq n+1$, the notation $a(\alpha, \beta ; P)$ means any plane on the point $P$ and on the $(n-1)$ points $A i$ with $i \neq \alpha, i \neq \beta$. We use also the dual notation $A(\alpha, \beta ; p)$.

Lemma D2. Under the above hypotheses, at least one plane $b=a(\alpha, \beta ; P)$ exists, and any sucb plane satisfies eitber $A \alpha \sim b$ or $A \beta \sim b$.

Proof. Trivial consequence of Axiom 1 and Lemma C. $\square$

Lemma E2. Given a simplex $\mathfrak{A}$, a plane $r$ and two integers $\beta, \gamma$ with $1 \leq \beta<$ $\gamma \leq(n+1)$, there exists a simplex $\mathcal{C}$ such that either $C \beta \circ r$ or $C \gamma \circ r$ and sucb that $C i=A i, c i=a i$ for all $i \neq \beta, i \neq \gamma$.

Proof. We consider the point $B=A(\beta, \gamma ; r)$ and the plane $b=a(\beta, \gamma ; B)$. Using Lemmas $C$ and $C$ dual, there are four cases:

Case 1. $B \sim a \beta, B \circ a \gamma$.

Case 2. $B \circ a \beta, B \sim a \gamma$.

Case 3. $B \sim a \beta, B \sim a \gamma, A \gamma \sim b$.

Case 4. $B \sim a \beta, B \sim a \gamma, A \beta \sim b, A \gamma \circ b$.

In Case 1 we obtain $\mathcal{C}$ from $\mathcal{Q}$ by replacing $A \beta$ by $B$. In Case 3 we obtain $\mathcal{C}$ from $\mathcal{A}$ by replacing $A \beta$ and $a \gamma$ by $B$ and $b$. Cases 2, 4 differ from Cases 1, 3 only by an interchange of $\beta$ and $\gamma$.

Lemma F2. Given a simplex $\mathbb{P}$ and a plane $r$ there exists a simplex $\mathfrak{D}$ such that $d(n+1)=r$. Moreover $D i=A i$, di=ai for all $i$ such that $A i \circ r, 1 \leq i \leq n$.

Lemma G2. And if $A(n+1) \sim r$ then $D(n+1)=A(n+1)$.

(Incidentally, Axioms 1, 2, 7N, $8 \mathrm{~N}$ imply Lemma $\mathrm{F}$ but not G.)

Proof of Lemma F. We consider three cases:

Case 5. $A i \sim r$ only for $i=n+1$.

Case 6. $A i \sim r$ only for $i=\beta$ where $1 \leq \beta \leq n$.

Case 7. $A i \sim r$ for at least two values of $i$; say $i=\beta, i=\gamma$.

In Case 5 we obtain $\mathfrak{D}$ from $\mathfrak{Q}$ by replacing $a(n+1)$ by $r$. In Case 6 we obtain $\mathscr{D}$ from $\mathbb{A}$ by replacing $A \beta, A(n+1), a \beta, a(n+1)$ by $A(n+1), A \beta, a(n+1), r$ respectively. In Case 7 we apply Lemma $E$ repeatedly, until we get out of this case.

Proof of Lemma G. Let $\beta, \gamma$ be the smallest values of $i$ such that $A i \sim r$. We consider three cases:

Case 8. $\beta=n+1, \gamma$ does not exist.

Case 9. $\beta<y=n+1$.

Case 10. $\beta<\gamma \leq n$. 
Then Case 8 is the same as Case 5. In Case 9 let $B=A(\beta, n+1 ; r)$. Then $B \sim a \beta$ by Axiom $6 W$ with $T=B, t=a \beta, S N n=\{A 1 \uparrow A(\beta-1), A(\beta+1) \uparrow A(n+1)$; $a 1 \uparrow a(\beta-1), a(\beta+1) \uparrow a n, r\}$, or by Axiom $5 N$ with $A(n+1), B$ distinguished by $r$. Thus, similarly to Cases 1 and 3, the desired simplex is

$$
\mathcal{D}=\{A 1 \uparrow A(\beta-1), B, A(\beta+1) \uparrow A(n+1) ; a 1 \uparrow a n, r\} \text {. }
$$

In Case 10 we apply Lemma $F$ repeatedly until we get into Case 8 or 9.

Lemma H2. For any point $R$ there exists a simplex $\mathcal{E}$ such that $E(n+1)=R$.

Proof. We use the simplex $\mathfrak{C}$ of Axiom $3 S N$ and apply the dual of Lemma F.

Proof of Theorem A2. We apply Lemmas F, G to the simplex of Lemma H.

3. Reductions for $W$ and $S N$ spaces. We prove Theorems $A 1$ and the equivalence $R N \Leftrightarrow S N$ in Theorem B1. For a $W$-space which is not an $N$-space, see Example A9.

We use the notations $z$ (=zero), $y, n$ or $m$ affixed to axioms to indicate that the axioms hold for zero dimension, for one dimension, for the original (n-dimensional) space or for all reductions (they are $m=n-1$ dimensional) of the original space respectively.

Lemma A3. Axiom $1 n \Leftrightarrow$ Axiom $1 \mathrm{~m}$.

Proof. The implication Axiom $1 n \Rightarrow$ Axiom $1 m$ is trivial. For the converse we make an induction on $\alpha$, with $1 \leq \alpha \leq n$, in the statement "on every $\alpha$ points there exists a plane". Given $\alpha$ points $\{P 1 \uparrow P \alpha\}$, by the inductive hypothes is there exists a plane $p$ on $\{P 1 \uparrow P(a-1)\}$. Either $P \alpha \circ p$ and $p$ is as desired, or $P \alpha \sim p$, but then the reduction $[P \alpha, p]$ contains a plane $q$ on $\{P 1 \uparrow P(\alpha-1)\}$, and this plane $q$ is as desired.

Lemma B3. Axioms (3SNm, $\mathrm{cW}$ or $\mathrm{c}) \Rightarrow$ Axiom $3 S \mathrm{Nn}$. Conversely Axioms $\{\mathrm{SWn}\}$ or $\{\mathrm{SNn}\} \Rightarrow$ Axiom $3 \mathrm{SNm}$.

Proof. The first implication is trivial. The second implication results from Theorem A2.

Lemma C3. Axiom $6 W n \Leftrightarrow$ Axiom $6 W m$.

Proof. The singlenot $n$-square $\mathbb{A}$ in Axiom $6 W n$ and the singlenot $m$-square $B$ in Axiom $6 W m$ are related by $\left\{A 1 \uparrow A n, a 1 \uparrow a_{n}\right\}=\{B 1 \uparrow B(n-1), R ; b 1 \uparrow b(n-1)$, $r\}$.

Lemma D3. Axiom $4 \mathrm{Nn} \Leftrightarrow$ Axiom $4 \mathrm{Nm}$.

Proof. The $n$-simplex $\mathbb{Q}$ in Axiom $4 N n$ is related to the $m$-simplex $\mathfrak{B}$ in Axiom $4 N m$ by $\{A 1 \uparrow A(n+1) ; a 1 \uparrow a(n+1)\}=\{B 1 \uparrow B(n-1), R, B n ; b 1 \uparrow b(n-1), r, b n\}$.

Lemma E3. Axioms $(a W, b W) \Leftrightarrow A x i o m s\{S W z\}$. 
Proof. Axiom $a W=$ Axiom $6 W z$. Axiom $b W=$ Axiom $3 S N z$. Axiom $b W \Rightarrow A x-$ ioms $(1 z, 2 z)$.

Lemma F3. Axioms $(a, b N) \Leftrightarrow$ Axioms $\{S N y\}$.

Proof. Axiom $a \Rightarrow$ Axioms $(1,2,4 N, 5 N)$. Axioms $(a, b N) \Rightarrow$ Axiom $3 S N$. (Lemma $\mathrm{H} 2, \mathrm{Axiom} 4 N) \Rightarrow$ (at most one plane on each point). (This, its dual, Axioms 1, 2) $\Rightarrow$ Axiom $a$. Axiom $3 S N \Rightarrow$ Axiom $b N$.

Theorem G3. Axioms $\{S W\} \Leftrightarrow$ Axioms $\{R W\}$.

Proof. Use $(3 S N \Rightarrow \mathrm{c})$, Lemmas A, A dual, B, C, E.

Theorem H3. Axioms $\{S N\} \Leftrightarrow$ Axioms $\{R N\}$.

Proof. Use $(3 S N \Rightarrow c)$, Lemmas $A, A$ dual, $B, D, F$.

4. The $S N, T N$ equivalence. To prove this equivalence we use lemmas, including a Theorem $\mathrm{D} 4$ which requires the moving of a simplex.

Lemma A4. Axioms $\{T N\} \Rightarrow$ Axiom $3 S N$.

Proof. Let $\mathfrak{Q}$ be the semisimplex of Axiom $3 T N$ and let $A(n+1)$ be a point on the $n$ planes of $\mathbb{Q}$. For each integer $\alpha$ with $1 \leq \alpha \leq n+1$, let $b a$ be a plane on all points $A i$ except possibly $A \alpha$. Then $A \alpha \sim b a$ (Axiom $6 T)$. The simplex $\mathbb{B}=$ $\{A 1 \uparrow A(n+1) ; b 1 \uparrow b(n+1)\}$ satisfies Axiom $3 S N$.

Lemma B4. Axioms $\{T N\} \Rightarrow$ Axiom $4 N$.

Proof. Axiom $6 T \Rightarrow$ (no distinguishable planes are on the first $n$ points of a simplex). Hence $(4,6 T) \Rightarrow 4 N$.

Lemma C4. Axioms 1 and $4 N$ imply: Given a simplex $D$ and a point $R$ with $R \sim d 1$, there exists a simplex $B$ such that $B 1=R, B i=D i$ for $2 \leq i \leq n+1$, $b 1=d 1$.

Proof. For each integer $\alpha$ with $2 \leq a \leq n+1$ we define $b a=d(1, \alpha ; R)$. Then $D \alpha \sim b \alpha$ because otherwise $d 1$ and $b \alpha$ would be two distinct (because distinguished by $R$ ) planes on the last $n$ points of the simplex $\mathcal{D}$, contrary to Axiom $4 N$. The, desired simplex is $\mathbb{B}=\{R, D 2 \uparrow D(n+1) ; d 1, b 2 \uparrow b(n+1)\}$.

Theorem D4. Axioms $\{S N\}$ imply: For every singlenot a-triangle $\mathbb{A}$ (the plane aa may be missing) there exists a simplex $B$ such that $B i=A i$ for $1 \leq i \leq \alpha$.

Proof. We make an induction on $\alpha$. For $\alpha=1$ the theorem is true, by Lemma H2. For $a>1$ the inductive hypothesis and a circular permutation gives a simplex $C$ such that $C i=A i$ for $2 \leq i \leq a$. Lemmas $\mathrm{B} 2, \mathrm{~F} 2$ applied to $C$ and $r=a 1$ give a simplex $\mathscr{D}$ such that $d 1=a 1$ and $D i=C i$ for $2 \leq i \leq \alpha$. Lemma $C$ with $R=$ $A 1$ gives the desired simplex $\mathfrak{B}$. 
Lemma E4. Axioms $\{S N\} \Rightarrow$ Axiom $6 T$.

Proof. Let $T, t, T N n=\{A 1 \uparrow A n ; a 1 \uparrow a n\}$ be as in Axiom 6T. By Theorem D there exists a simplex $\mathscr{B}$ such that $\{B 1 \uparrow B(n+1)\}=\{A 1 \uparrow A n, T\}$. Then $t=b(n+1)$ (Axiom $4 N$ ), hence $T \sim t$.

Lemma F4. Axioms $\{S N\} \Rightarrow$ Axiom 4 .

Proof. Let $r, s$ be two distinct planes. There exists a simplex $E$ with $e(n+1)=$ $r$ (Lemma $\mathrm{H} 2$ dual). At least one of the points $\{E 1 \uparrow E n\}$ is not on $s$ (Axiom $4 N$ ), hence distinguishes $r, s$.

The orem G4. Axioms $\{S N\} \Leftrightarrow$ Axioms $\{T N\}$.

Proof. Use Lemmas A, B, B dual, E, F, F dual.

5. The nondual theory. We have discussed the Axioms $\{S N\}$ in an earlier publication [2], and gave there a self-dual treatment of flats, but we did not go far enough to compare Axioms $\{S N\}$ and $\{L N\}$. We do it now. We abandon our empha$s$ is on self-duality. The present results are not used in later sections.

We assume Axioms $\{S N\}$. A set-plane $p$ is defined as the set of all points on the plane $p$. A line is defined as the intersection of all set-planes containing two distinct points. If the se points are $P, Q$, the line is denoted by $\overline{P Q}$.

Lemma A5. In any simplex $\mathfrak{A}$, the line $\overline{A 1 A 2}$ is the intersection of the planes $\{a 3 \uparrow a(n+1)\}$.

Proof. The two points $P=A 1, Q=A 2$, and therefore all points of $\overline{P Q}$, are on $\{a\} \uparrow a(n+1)\}$. Conversely, if $R$ is a point on these planes, and if $r$ is a plane on the two points $P, Q$, then $R \circ r$, because otherwise we contradict Axiom $6 T$ with $T=Q, t=a 3, T N n=\{A 4 \uparrow A(n+1), R, P ; a 4 \uparrow a(n+1), r, a 1\}$. Thus $R$, being on all such planes $r$, is in $\overline{P Q}$.

Lemma B5. If $R, S$ are distinct points in a line $\overline{P Q}$, then $\overline{P Q}=\overline{R S}$.

Proof. There exists a simplex $\mathbb{A}$ with $A 1=P, A 2=Q$ (Theorem D4). We may assume $P \neq S$. Then $S \sim a 2($ Axiom $5 N)$. Let $s=a(1,2 ; S)$. Then $s \neq a 2$, hence $P \sim s$ (Axiom $4 N)$. Thus $B=\{P, S, A 3 \uparrow A(n+1) ; s, a 2 \uparrow a(n+1)\}$ is a simplex. Comparing the planes of $\mathbb{Q}$ and $\mathscr{B}$, we get $\overline{P Q}=\overline{P S}$ (Lemma $A$ ). Similarly $\overline{P S}=$ $\overline{R S}$.

Lemma C5. Lines and reductions to one dimension are identical.

Proof. Each line $\overline{P Q}$ is the set of points of the one-dimensional space obtained by the succession of the $(n-1)$ reductions $[A 3, a 3] \uparrow[A(n+1), a(n+1)]$, with $\mathbb{Q}$ defined as above. Conversely each reduction to one dimension has two points $P, Q($ Axiom $\mathrm{b} N)$ and is identical to $\overline{P Q}$. 
Lemma D5. Axioms $\{S N\} \Rightarrow$ Axioms (I, IIN, III).

Proof. The definition of a line and Lemma B imply Axioms I and IIN. Let $P$, $Q, R, S, T$ be defined as in Axiom III. Excluding the trivial case of five collinear points, there exists a singlenot 3-triangle with points $Q, S, P$, hence (Theorem D4) there exists a simplex $\mathbb{Q}$ with $A 1=Q, A 2=S, A\}=P$. Then $\mathbb{B}=\{R, T, P$, $A 4 \uparrow A(n+1) ; a 1, a 2, b 3, a 4\lceil a(n+1)\}$, for an appropriate plane $b 3$, also is a simplex. There exists a point $U$ on the $n$ planes $\{a 3, b 3, a 4 \uparrow a(n+1)\}$. This point $U$ is in $\overline{Q S}$ and in $\overline{R T}$, as shown by $\mathbb{Q}$ and $\mathfrak{B}$. This proves Axiom III.

In any plane satisfying Axioms (I, IIN, III) Dembowski defines: A set $s$ of points is a subspace if for any two distinct points $P, Q$ in $s$ every point of the line $\overline{P Q}$ is in $s$. A point $A$ is independent of a set $\delta$ if there exists a subspace containing $\mathcal{S}$ but not $A$. A set $\mathcal{S}$ is independent if every point in $\mathcal{S}$ is independent of the remaining points in $\mathcal{S}$.

Lemma E5. Axioms $\{S N\} \Rightarrow$ Axiom IV.

Proof. Let $\mathbb{Q}$ be the simplex of Axiom $3 S N$. For each integer $a$ with $1 \leq \alpha \leq$ $n+1$ the point $A \alpha$ is independent of the other points of $\mathbb{A}$ because $a \alpha$ is a subspace containing these other points but not $A \alpha$.

Lemma F5. For any simplex $\mathbb{Q}$ and point $R$, the point $R$ depends on $\{A 1 \uparrow$ $A(n+1)\}$.

Proof. We move a simplex $\mathcal{B}$ from the initial position $\mathbb{Q}$ into a final position $\mathcal{E}$ such that $E(n+1)=R($ Lemma $\mathrm{H} 2)$. Each new point introduced during this motion has the form $C=B(\beta, \gamma ; r)$, hence is on $\overline{B \beta B \gamma}$ (Lemma $A)$, thus dependent on $\{B 1 \uparrow B(n+1)\}$. In particular $R$ is dependent on $\{B 1 \uparrow B(n+1)\}$ and each $B \alpha$ is dependent on $\{A 1 \uparrow A(n+1)\}$.

Lemma G5. Axioms $\{S N\} \Rightarrow$ Axiom V.

Proof. We make an induction on dimensions, by proving that Axioms ( $\{S N\}$, $V m\}) \Rightarrow$ Axiom $V n$. Given $(n+2)$ points $\{A 1 \uparrow A(n+2)\}$, if $\{A 1 \uparrow A(n+1)\}$ are independent then they are not coplanar (Axiom $V m$ ), hence they form a simplex, and $A(n+2)$ depends on them (Lemma F). This establishes Axiom $V n$.

Dembowski defines a hyperplane as a maximal proper subspace.

Lemma H5. The points and byperplanes of any space LN satisfy Axioms \{SN\}.

We do not prove this well-known result.

Theorem J5. Axioms $\{L N\} \Leftrightarrow$ Axioms $\{S N\}$ and Axioms $\{L P\} \Leftrightarrow$ Axioms $\{R P\}$.

Proof. The first $\Leftrightarrow$ holds by Lemmas D, E, G, H. The second $\Leftrightarrow$ holds by Theorem $\mathrm{H} 3$ and because Axiom II $P \Leftrightarrow$ Axiom $b P$ (Lemma C). 
Incidentally, the following theorem is true for simplicial spaces, but not for weak spaces.

Theorem K5. Any two reductions $[R, r]$ and $[S, r]$ for the same plane $r$ consist of the same point and the same set-planes.

We may denote this reduction by $[r]$. See Example A9.

6. The $R P \Rightarrow T P \Rightarrow D P$ implications. We prove

Theorem A6. The sets of axioms satisfy $\{R P\} \Rightarrow(\{S N\}, 3 S P) \Rightarrow\{T P\} \Rightarrow\{T U\} \Rightarrow$ $\{S U\} \Rightarrow\{D U\}$.

We use several lemmas.

Lemma B6. Axioms $\{R P\} \Rightarrow$ Axiom $3 S P$.

Proof. We may use Axioms $\{S N\}$ (by $R P \Rightarrow R N$ and Theorem H3). Let $\mathbb{Q}$ be the simplex of Axiom $3 S N$. The succession of the $(n-1)$ reductions $[A 1, a 1] \uparrow$ $[A(n-1), a(n-1)]$ is a one-dimensional space containing the two points $A n$, $A(n+1)$ and (Axiom $b P)$ a third point $P$. Then $P \neq A(n+1)$ and Axiom $5 N$ gives $P \sim a n$, while $P \neq A n$ gives $P \sim a(n+1)$. Thus we have found a point $B n=P$ satisfying $B n \circ a j$ for and only for $j \neq n, j \neq n+1$. Similarly, for each integer $\alpha$ with $1 \leq \alpha<n$, after a permutation (Lemma B2), we can find a point $B \alpha$ satisfying $B \alpha \circ a j$ for and only for $j \neq a, j \neq a+1$. Then $B=\{B 1 \uparrow B n, A(n+1) ; a 1 \uparrow a(n+1)\}$ satisfies Axiom $3 S P$.

Lemma C6. Axioms $\{T U\} \Rightarrow$ Axiom $3 S P$.

Proof. Let $\mathbb{A}$ be the semidoublex Axiom 3TP. We construct a doublenot square $\mathcal{C}$ with the same points as $\mathcal{Q}$. For each integer $\alpha$ with $3 \leq \alpha \leq n+1$, let $B a$ be a point on the $n$ planes $a j$ with $j \neq \alpha-1$, and let $c \alpha=a(\alpha-1, \alpha ; B \alpha)$ be a plane on the one point $B a$ and on the $(n-1)$ points $A i$ with $i \neq \alpha-1, i \neq \alpha$. The point $B \alpha$ and the plane $c \alpha$ exist. Moreover $A(\alpha-1) \sim c \alpha$ and $A \alpha \sim c \alpha$ because otherwise we would contradict Axiom $6 T$ by taking $T=B \alpha, t=c \alpha, T N n=\{A 1 \uparrow$ $A(\alpha-2)$, either $A(\alpha-1)$ or $A \alpha, A(\alpha+1) \uparrow A(n+1)$; a1 $\uparrow a(\alpha-2)$, a $a \uparrow a(n+1)\}$. Then $C=\{A 1 \uparrow A(n+1) ; a 1, a 2, c 3 \uparrow c(n+1)\}$ satisfies Axiom $3 S P$.

Lemma D6. Axioms $\{S U\} \Rightarrow$ Axiom $3 D$.

Proof. Let $A$ be the doublenot square of Axiom 3SP. Let $b$ be a plane on $\{A 1 \uparrow A n\}$ and $B$ be a point on $\{a 2 \uparrow a(n+1)\}$. Then $A(n+1) \sim b$ and $B \sim a 1$, by Axioms $(7 P, 8 P)$ respectively. Also $B \sim b$ by Axiom $7 P$ with $S P(n+1)=\{A n \downarrow$ $A 1, B ; a(n+1) \downarrow a 1\}$. The desired doublex is $\{A 1 \uparrow A(n+1), B ; a 1 \uparrow a(n+1), b\}$.

Proof of Theorem A6. It is obvious that $\{R P\} \Rightarrow\{R N\}, 3 S P \Rightarrow 3 T P,\{T P\} \Rightarrow$ $\{T U\}, 6 T \Rightarrow(7 P, 8 P) \Rightarrow(6 D, 7 D, 8 D)$. The other needed results are Theorems $\mathrm{H} 3$, G4 and Lemmas B6, C6, D6.

7. Moving a doublex. We assume Axioms $\{D U\}$ and prove: 
Theorem A7. Every doublenot a-square $\mathbb{A}$ (or $\mathbb{Q}$ plus a point $A(\alpha+1)$ on $\{a 1 \uparrow$ aa\}) can be completed into a doublex.

Integers are taken in the additive algebra modulus $(n+2)$. The inequality $\alpha<\beta$ means $1 \leq \alpha<\beta \leq n+2$, and the arrows $\uparrow$ or $\downarrow$ mean in the increasing or decreasing cyclical order, starting with the term on the left and ending with the term on the right. Thus for $n=4$ we have

$$
\begin{array}{ll}
\{A 2 \uparrow A 4\}=\{A 2, A 3, A 4\}, & \{A 2 \downarrow A 4\}=\{A 2, A 1, A 6, A 5, A 4\}, \\
\{A 4 \uparrow A 2\}=\{A 4, A 5, A 6, A 1, A 2\}, & \{A 4 \downarrow A 2\}=\{A 4, A 3, A 2\} .
\end{array}
$$

When $\alpha=\beta+1$ the context will make it clear whether $\{A \alpha \uparrow A \beta\}$ represents the empty set or the set of all $A i$.

Lemma B7. Any doublex $\mathbb{Q}$ and plane $r$ satisfy $A i \sim r$ for at least two values of $i$.

Proof. Otherwise there would exist an integer $a$ such that $A i \circ r$ for $i \neq a$. Then $A \alpha{ }^{\circ} r$ is contradicted by Axiom $7 D$, and $A \alpha \sim r$ is contradicted by Axiom $6 D$ with $S P(n+2)=\{A a \uparrow A(\alpha-1) ; r, a(\alpha+1) \uparrow a(\alpha-1)\}$.

Given a doublex $\mathfrak{Q}$ and two distinct integers $\alpha, \beta$, the notation $a(\alpha, \beta)$ means any plane on the $n$ points $A i$ with $i \neq \alpha, i \neq \beta$. Given a doublex $\mathbb{A}$, a point $B$, and three distinct integers $\alpha, \beta, \gamma$, the notation $a(\alpha, \beta, \gamma ; B)$ means any plane on the point $B$ and on the $(n-1)$ planes $A i$ with $i \neq \alpha, i \neq \beta, i \neq \gamma$.

Given a doublex $\mathfrak{A}$ and two distinct integers $\alpha, \gamma$, a permutation of planes gives (Lemma $C$, below) a new doublex $\mathfrak{B}=\mathfrak{A}(B \alpha, B y)$ defined by

$$
B \alpha=A(\alpha, \gamma), \quad B \gamma=A(\alpha+1, \gamma+1),
$$

$\{B(\gamma+1) \uparrow B(\alpha-1)\}=\{A(\gamma+1) \uparrow A(\alpha+1)\}, \quad\{B(\alpha+1) \uparrow B(\gamma-1)\}=\{A(\gamma-1) \downarrow A(\alpha+1)\}$,

$$
\{b(\gamma+1) \uparrow b a\}=\{a(\gamma+1) \uparrow a \alpha\}, \quad\{b(\alpha+1) \uparrow b \gamma\}=\{a \gamma \downarrow a(a+1)\} .
$$

If for the point $B a$ or $B \gamma$ we can use a previously defined point $C$ then we write $\mathfrak{Q}(B \alpha=C, B \gamma)$ or $\mathfrak{Q}(B \alpha, B \gamma=C)$.

Given a doublex $\mathbb{A}$, a point $B$ and two integers $\beta, \gamma$ such that $\gamma+1 \neq \beta \neq \gamma$, $B \sim a \gamma, B \sim a(\gamma+1)$, but $B \circ a j$ for $j \neq \beta, j \neq \gamma, j \neq \gamma+1$, we obtain (Lemma $C$, below) a new doublex $\mathcal{C}=\mathbb{A}(C \gamma=B, c \beta)$ defined by

$$
\begin{aligned}
& C \gamma=B, \quad c \beta=a(\beta-1, \beta, \gamma ; B), \\
& C i=A i \quad \text { for } i \neq \gamma, \quad c j=a j \quad \text { for } j \neq \beta .
\end{aligned}
$$

The notations $A(\alpha, \beta), A(\alpha, \beta, \gamma ; b), \mathfrak{A}(b \alpha, b \gamma), \mathfrak{A}(c \beta, c \gamma=b)$ are defined by duality.

Lemma C7. The two $(n+2)$-squares $\mathfrak{B}=\mathfrak{A}(B \alpha, B \gamma), \mathcal{C}=\mathbb{A}(C \gamma=B, c \beta)$ defined above exist and are doublexes. 
Proof. The points $B \alpha, B y$ exist (Axiom 2) and satisfy the needed $\sim$ (Lemma $\mathrm{B}$ dual). The plane $c \beta$ exists (Axiom 1 ), and satisfies $A(\beta-1) \sim c \beta, A \beta \sim c \beta$, as we show by elimintaing the other alternatives: $A(\beta-1) \circ c \beta, A \beta \circ c \beta$ is contradicted by Lemma B. $A(\beta-1) \circ c \beta, A \beta \sim c \beta$ is contradicted by Axiom $6 D$ with $S P(n+2)=\{C \beta \uparrow C(\beta-1) ; c \beta \uparrow c(\beta-1)\} . A(\beta-1) \sim c \beta, A \beta \circ c \beta$ is contradicted by Axiom $6 D$ with $S P(n+2)=\{C(\beta-1) \downarrow C \beta ; c \beta \downarrow c(\beta+1)\}$.

Given a doublex $\mathbb{Q}$ and a plane $r$, we denote by $\beta(\mathfrak{Q}), \gamma(\mathfrak{A})$, with $\beta<\gamma$, the largest two integers $i$ such that $A i \sim r$.

Lemma D7. Given a doublex $\mathbb{Q}$ and a plane $r$, let $\beta=\beta(\mathfrak{Q})$ and assume $\beta \geq 2$. Then there exists a doublex $\mathfrak{B}$ satisfying $\beta(\mathfrak{B})<\beta(\mathfrak{A}), \gamma(\mathfrak{B}) \leq \gamma(\mathfrak{Q}), B i=A$ i for $1 \leq i<\beta-1, b j=a j$ for $1 \leq j<\beta$.

Lemma E7. Moreover either $\gamma(\mathfrak{B})<\gamma(\mathfrak{Q})$ or $B(\beta-1)=A(\beta-1)$.

Proofs. Let $\gamma=\gamma(\mathfrak{Q}), B=A(\beta, \gamma, \gamma+1 ; r)$. We consider three cases.

Case 1. $B \sim a \gamma, B \sim a(\gamma+1)$,

Case 2. $B \sim a \beta, B \sim a \gamma, B \circ a(\gamma+1)$,

Case 3. $B \sim a \beta, B \circ a \gamma, B \sim a(\gamma+1)$.

The doublex $\mathcal{B}$ satisfying Lemma $D$ is

Case 1. $\mathfrak{B}=\mathfrak{A}(B y=B, b \beta)$,

Case 2. $\mathfrak{B}=\mathfrak{A}(B \beta=B, B \gamma)$,

Case 3. $\mathfrak{B}=\mathbb{A}(B(\beta-1), B \gamma=B)$.

Moreover we get $\gamma(\mathfrak{B})<\gamma(\mathfrak{Q})$ in Cases 1 and 3 , and we get $B i=A i$ for $1 \leq i<\beta$ in Cases 1 and 2.

Lemma F7. Given a doublex $\mathbb{Q}$, a plane $r$ and an integer $\delta$, there exists a doublex $\mathcal{C}$ such that $\beta(\mathcal{C}) \leq \delta, C i=A i$ for $1 \leq i<\delta$, cj=aj for $1 \leq j \leq \delta$.

Lemma G7. Moreover eitber $\gamma(\mathcal{C})<n+2$ or $C \delta=A \delta$.

Proofs. If $\beta(\mathfrak{Q})>\delta$ we apply Lemma $\mathrm{D}$ repeatedly until we get a doublex $\mathcal{C}$ with $\beta(\mathcal{C}) \leq \delta$. This proves Lemma F. Moreover Lemma E implies Lemma G.

Lemma H7. Given a doublex $\mathbb{A}$, a plane $r$ and an integer a sucb that $A i \circ r$ for $1 \leq i<\alpha$, there exists a doublex $D$ such that $d(\alpha+1)=r, D i=A i$ for $1 \leq i<\alpha$, $d j=a j$ for $2 \leq j \leq \alpha$.

Lemma J7. Moreover if $A \alpha \sim r$ then $D \alpha=A \alpha$.

Lemma K7. Or if $A(\alpha+1) \sim r$ then $d 1=a 1$.

In the following three proofs, let $\beta=\beta(\mathcal{C}), \gamma=\gamma(\mathcal{C})$.

Proof of Lemma H. Let $\mathcal{C}$ be the doublex of Lemma $\mathrm{F}$ for $\delta=\alpha$. Then $\beta=\alpha$, $r=c(\alpha, \gamma)$. The desired doublex is $\mathfrak{D}=\mathcal{C}(d(\alpha+1)=r, d(\gamma+1))$. 
Proof of Lemma J. Let $\mathcal{C}$ be the doublex of Lemma $\mathbf{F}$ for $\delta=\alpha+1$. If $\beta=\alpha$ the desired doublex $\mathscr{D}$ is as defined in the proof of Lemma $H$. If $\beta=\alpha+1$ then the desired doublex is $\mathfrak{D}=\mathcal{C}(D y, d(\alpha+1)=r)$.

Proof of Lemma K. If $A \alpha \circ r$ we look at the proof of Lemma H. We have $C \alpha \sim r$, hence $C \alpha \neq A \alpha$, and Lemma G gives $\gamma<n+2$. Then $\gamma+1 \neq 1$ and $d 1=$ $c 1=a 1$. If $A \alpha \sim r$ we look at the proof of Lemma J. Either $C(\alpha+1) \circ r$, then $C(\alpha+1) \neq A(\alpha+1)$, hence again $\gamma<n+2$, or $C(\alpha+1) \sim r$, then $d j=c j$ for $j \neq \gamma$.

Lemma L7. Given a doublex $\mathcal{E}$, a point $R$ and an integer a such that $R \circ e^{j}$ for $1 \leq j<\alpha$, there exists a doublex $\mathcal{F}$ such that $F \alpha=R, F i=E i$ and $f i=e i$ for $1 \leq i<\alpha$.

Lemma M7. Moreover if $R \sim$ ea then $f \alpha=e \alpha$.

Proofs. These two lemmas are obtained from Lemmas $\mathrm{H}$, J by the duality $A i \rightarrow e i, a(i+1) \rightarrow E i, r \rightarrow R$.

Lemma N7. For any point $R$ and plane $r$ with $R \sim r$ there exists a doublex $D$ with $D 1=R, d 1=r$.

Proof. To the doublex $\mathcal{E}$ of Axiom $3 D$ we apply Lemma $\mathrm{L}$ with $\alpha=1$ to obtain a doublex $\mathcal{F}$ with $F 1=R$, and to the doublex $\mathcal{F}$ we apply Lemmas $\mathrm{H}$, J with $a=1$ to obtain the desired doublex $\mathscr{D}$.

Proof of Theorem 17. We refer to this theorem by $A \alpha$ without the parentheses and by $A \alpha+$ with the parentheses. We make an induction on $\alpha$. Theorem $A 1$ is true, being the same as Lemma $\mathrm{N}$. Now we assume Theorem $A(\beta-1)$. Let $\mathbb{A}$ be a doublenot $\beta$-square, and let $i$ mean all $i$ in $1 \leq i<\beta$. The inductive hypothesis gives a doublex $\mathcal{B}$ with $B i=A i, b i=a i$. Lemma $\mathrm{L}$ for $\alpha=\beta$ gives a doublex $\mathcal{C}$ with $C i=B i, C \beta=A \beta, c i=b i$. Lemmas $\mathrm{H}, \mathrm{J}, \mathrm{K}$ for $\alpha=\beta-1$ give a doublex $\mathscr{D}$ with $D i=C i, d i=c i, d \beta=a \beta$ (but $D \beta \neq A \beta$ ). Lemmas $\mathrm{L}, \mathrm{M}$ for $\alpha=\beta$ give a

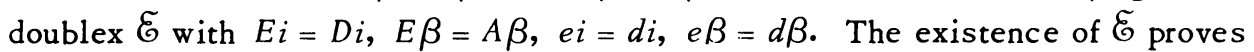
Theorem $A \beta$. This completes the induction. The existence of $\mathcal{C}$ proves Theorem $A \alpha+$.

8. The $D P \Rightarrow R P$ implications. We prove

Theorem A8. Axioms $\{D P\} \Rightarrow$ Axioms $\{R P\}$.

Theorem B8. Axioms $\{D U\} \Rightarrow$ Axioms $6 T$.

We use most previous results and additional lemmas.

Lemma C8. Axioms $\{D U\} \Rightarrow$ Axiom $6 P$.

Proof. The doublenot $n$-square $\mathbb{Q}=S P n$ plus the point $A(n+1)=T$ of Axiom GP can be completed into a doublex $B$ (Theorem A7). Hence $T \sim t$ (Lemma B7). 
We recall that $n$ means for the original space, $m$ means for all reductions $[R, r]$, and $y$ means for one dimension. We assume $n \geq 2$.

Lemma D8. Axiom $6 P_{n} \Rightarrow$ Axioms $(6 D m, 7 D m, 8 D m)$.

Proof. The $S P(m+2)=\{A 1 \uparrow A(n+1) ; a 1 \uparrow a(n+1)\}$ in Axiom $6 D m$ cannot exist, by Axiom $6 P_{n}$ with $T=A(n+1), t=r, S P_{n}=\{A 1\lceil A n ; a 1 \uparrow a n\}$. A plane $p$ in $[R, r]$ on the $m$-doublex $\mathscr{D}$ is Axiom $7 D m$ cannot exist, by Axiom $6 P n$ with $T=$ $R, t=p, S P_{n}=\{D 1 \uparrow D n, d 1 \uparrow d n\}$. Duality gives Axiom $8 D m$.

Lemma E8. Axioms $\{D U n\} \Rightarrow$ Axiom $3 D m$.

Proof. Given $R, r$ with $R \sim r$ there exists a doublex $\mathbb{Q}$ with $A 1=R, a 1=r$ (Lemma N7). Let $B=A(2, n+2), b=a(2, n+2)$. The desired $m$-doublex is $\{A 2 \uparrow$ $A(n+1), B ; b, a 3 \uparrow a(n+2)\}$, noticing that $B \sim b$ by Axiom $6 D m$.

Lemma F8. Axioms $\left\{D P_{n}\right\} \Rightarrow$ Axiom $5 m$.

Proof. Let $P, Q$ be two distinct points in a reduction $[R, r]$, where distinct means distinguishable by a plane $s$ in the original space (Axiom $5 n$ ). The case $R \circ s$ being trivial, we assume $R \sim s$. Then the doublenot rectangle $\{R, P, Q$; $r, s\}$ can be completed into a doublex $\mathcal{B}$ (Theorem A7). The plane $b 4$ is in $[R, r]$ and distinguishes $P, Q$.

Lemma G8. Axioms $\{D P y\} \Rightarrow$ Axioms $(a, b P)$.

Proof. Lemma $C$ for $n=1$ becomes $\{D U y\} \Rightarrow 6 T y$. We have also $3 D \Rightarrow 3 T N$. Hence $\{D P y\} \Rightarrow\{T N y\} \Rightarrow(a, b N)$. Moreover $3 D y \Rightarrow b P$.

Proof of Theorems A8 and B8. We get Theorem A8 from Lemmas C, D, E, F, $\mathrm{F}$ dual, G. We get Theorem B8 from Theorems A8, H3, E4 and by defining distinct to mean distinguishable in the original space.

9. Counterexamples and weaker axioms. First we give one example of a nonsimplicial weak space and two examples of nonprojective simplicial spaces.

Example A9. We consider the space of five points and five planes with the following incidence table

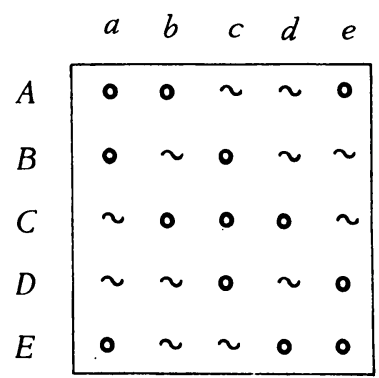

This space satisfies Axioms $\{S W\}$ for $n=2$, but not Axiom $6 T$ (take $T=A, t=a$, 
$T N 2=\{B, E ; e, b\})$. The distinguishable points $A, E$ are not distinguishable in the reduction $[B, e]$. The reduction $[B, e]$ satisfies neither Axiom $3 T P$ nor Axiom 5 , while the reduction $[C, e]$ is projective. Thus Theorem $\mathrm{K} 5$ fails.

Example B9. The space consisting of a simplex only is simplicial but not projective.

Example C9. Let $\mathcal{P}$ and 2 be two projective spaces. We declare that each point of $\mathcal{P}$ is on all planes of $\mathscr{2}$, and that each point of $\mathcal{Q}$ is on all planes of $\mathcal{P}$. We form thus a new space $\pi$, which we call the join of $\mathcal{P}$ and 2 . This space $\pi$ is a nonprojective simplicial space, with dimension $n=\alpha+\beta+1$, where $\alpha$ and $\beta$ are the dimensions of $\mathcal{P}$ and 2.

Proof. The join $\pi$ satisfies Axioms $(1,2,3 S N, 4,5,6 T)$, but the lines joining any one point of $\mathcal{P}$ to any one point of $\mathcal{Q}$ have only two points. Thus Axiom $\mathrm{b} P$ is not satisfied.

We say that an Axiom $A$ can be weakened within a set $\{A, B, C, \ldots\}$ of axioms if there exists an Axiom $A^{\prime}$, which does not imply $A$, such that $\left\{A^{\prime}, B, C, . .:\right\} \Rightarrow A$.

Theorem A9. Axiom 6T can be weakened within Axioms $\{T P\}$ by an additional incidence specification.

The added specification is $S 1 \sim s 2$ for the semisimplex $\delta$ in Axiom $6 T$.

Proof. Using this weaker Axiom 6 $T^{\prime}$, the proof of Lemma $C 6$ remains valid with $c 3=a(2,3 ; B 3)$ if $A 1 \sim a 3$ and with $c 3=a 3$ if $A 1 \circ a 3$. Thus we proved $A x-$ iom $3 S P$, hence Axioms $\{S U\},\{D U\}$ (Theorem A6) and Axioms $6 T$ (Theorem B8).

Theorem B9. Axiom 3TP cannot be weakened within Axioms $\{T P\}$ by deletion of an incidence specification.

Lemma C9. Given two integers $\alpha, \beta$ with $1 \leq \alpha<\beta \leq n+2$, Axioms $\{D U\}$ imply the existence of an $(n+2)$-square $B$ which is doublenot except that $B \beta \sim b a$.

Proof of Lemma C. Let $\mathbb{Q}$ be a doublex, let $P=A(1, \alpha+1), p=a(1, \beta)$. Then $P \sim p$ by Axiom $6 T$ (proved in Theorem B8) with $T=P, t=p, T P n=\{A 2 \uparrow A \alpha$, $A(\beta-1) \downarrow A(\alpha+1), A(\beta+1) \uparrow A(n+2) ; a 2 \uparrow a \alpha, a \beta \downarrow a(\alpha+2), a(\beta+1) \uparrow a(n+2)\}$. The desired $(n+2)$-square $B=\{A(\alpha-1) \downarrow A 1, P, A(\alpha+1) \uparrow A(n+2) ; a \alpha \downarrow a 2, p$, $a(\alpha+1) \uparrow a(n+2)\}$.

Proof of Theorem B. When $\mathcal{P}$ and $\mathcal{Q}$ are projective spaces or zero-dimensional weak spaces, then their join $\pi$ in Example $C$ satisfies the Axiom $3 S P^{\prime}$ obtained from Axiom $3 S P$ by deletion of any one of the three specifications $A(\alpha+1) \sim$ $a(\alpha+1), A(\alpha+1) \sim a(\alpha+2), A(\alpha+2) \sim a(\alpha+2)$. The Axiom 3SP" obtained from Axiom $3 S P$ by deletion of any one $A \beta \circ a \alpha$, for $1 \leq \alpha<\beta \leq n+1$, is satisfied by any $(n-1)$-dimensional projective space $\mathcal{P}$ (Lemma $C$ ), and hence by the join $\pi$ of $\mathcal{P}$ with a zero-dimensional weak space 2 . Thus neither Axioms ( $\left.\{T N\}, 3 S P^{\prime}\right)$ nor Axioms ( $\left.\{T N\}, 3 S P^{\prime \prime}\right)$ imply Axiom bP. 


\section{REFERENCES}

1. P. Dembowski, Finite geometries, Ergebnisse der Mathematik und ihrer Grenzgebiete, Band 44, Springer-Verlag, Berlin and New York, 1968. MR 38 \#1597.

2. M. Esser, Self-dual postulates for n-dimensional geometry, Duke Math. J. 18 (1951), 475-479. MR 13, 59.

3. W. M. Kantor, Characterizations of finite projective and affine spaces, Canad. J. Math. 21 (1969), 64-75. MR 38 \#4338.

4. D. Pedoe, An introduction to projective geometry, Internat. Series of Monographs on Pure and Appl. Math., vol. 33, Pergamon Press, Oxford, 1963. MR 33 \#600.

5. A. Winternitz, Zur Begründung der projektiven Geometrie: Einführung idealer Elemente unabhängig von der Anordnung, Ann. of Math. (2) 41 (1940), 365-390. MR 1, 260.

DEPARTMENT OF MATHEMATICS, UNIVERSITY OF DAYTON, DAYTON, OHIO 45409 